\title{
Einstein Manifolds, Abelian Instantons, Bundle Reduction, and the Cosmological Constant
}

\author{
Chopin Sod \\ Physics Department, National Cheng Kung University, \\ Tainan, Taiwan 70101, Taiwan.
}

\begin{abstract}
The anti-self-dual projection of the spin connections of certain four-dimensional Einstein manifolds can be Abelian in nature. These configurations signify bundle reductions. By a theorem of Kobayashi and Nomizu such a process is predicated on the existence of a covariantly constant field. It turns out that even without fundamental Higgs fields and other physical matter, gravitational self-interactions can generate this mechanism if the cosmological constant is non-vanishing. This article identifies the order parameter, and clarifies how these Abelian instanton solutions are associated with a Higgs triplet which causes the bundle reduction from $\mathrm{SO}(3)$ gauge group to $\mathrm{U}(1)$.
\end{abstract}

PACS numbers: 11.15.-q, 11.15.Ex, 04.20.Cv

\section{INTRODUCTION}

Recent Type Ia Supernovae observations strongly support a non-vanishing and positive cosmological constant [1]. In four-dimensions many exact and interesting solutions of Einstein's field equations $R_{\mu \nu}=\lambda g_{\mu \nu}$ with cosmological constant, $\lambda$, are known. With a non-vanishing cosmological constant the (anti)self-dual part of the spin connections of certain Einstein manifolds can be Abelian. Such a solution of Einstein's equations signify a bundle reduction. For this to happen a theorem of Kobayashi and Nomizu demands the existence of a covariantly constant field on an associated bundle [2]. The relation of this field to the cosmological constant in the bundle reduction process is clarified in this article. Although Kobayashi and Nomizu's theorem requires such fields for all bundle reductions, it sheds no further light on their precise nature. Indeed it remains to be seen whether current particle physics searches for Higgs fields responsible for symmetry breaking in the standard model will encounter fundamental or composite objects. Kobayashi and Nomizu's theorem however seems to guarantee their existence in the description of bundle reduction processes. It is therefore remarkable Abelian instantons discussed in this article demonstrate that with a non-vanishing cosmological constant gravitational self-interactions can generate the mechanism of bundle reduction dynamically, despite the absence of additional fundamental Higgs fields and other physical matter.

After an introduction on self and anti-self-dual decompositions of the Riemannian curvature in the next section a couple of explicit Abelian instantons configurations are recalled in Section III. The theorem of Kobayashi and Nomizu is recounted in Section IV, together with its specialization to bundle reduction of the $S O(3)$ gauge group to $U(1)$. The role of the cosmological constant is clarified in Section V, and the article ends with a note on Abelian instantons and their relation to Kähler-Einstein manifolds in Section VI.

\section{SELF AND ANTI-SELF-DUAL DECOMPOSITIONS OF THE RIEMANNIAN CURVATURE}

In contradistinction to $S O(3,1)$ for Lorentzian signature, the local Euclidean symmetry of the theory is gauged by an $S O(4)$ spin connection in the case of Euclidean signature discussed in this article. See, for instance, Ref. [3] on the importance of Euclidean instanton solutions in semi-classical and quantum gravity. Since $S O(4)=\left[S U(2) \times S U(2] / Z_{2}\right.$, the self and anti-self-dual parts of the spin connection are generically $S O(3)$ in value (in the Lorentzian instance $S O(3,1)$ is isomorphic to $S O(3, C)$, and self and anti-self-dual projections are related by complex conjugation). It is both natural and useful to analyze the properties of four-dimensional Einstein manifolds in terms of these projections of the spin connections. Four dimensions also has the peculiarity of allowing a decomposition of the Riemann curvature two-form into components taking values in the $( \pm 1)$ eigenspaces, $\Lambda_{2}^{ \pm}$, of the Hodge duality operator $*$. To be precise, the bases for $\Lambda_{2}^{ \pm}$can regarded to be the self and anti-self-dual two-forms $\Sigma_{a}^{ \pm} \equiv \pm e_{0} \wedge e_{a}+\frac{1}{2} \epsilon_{0 a b c} e^{b} \wedge e^{c}$ with $e_{A}$ denoting the vierbein. Explicitly $* \Sigma^{ \pm}= \pm \Sigma^{ \pm}$. The conventions are that upper case Latin Euclidean Lorentz indices take values from 0 to 3 , while lower case indices run from 1 to 3 . Spacetime components are represented by Greek indices.

The Riemann-Christofel curvature tensor $R_{A B C D}$ has four indices (anti-symmetric in pairs), and can be dualized both on the left and on the right. These can be thought of as internal and external duality transformations if one considers the Riemann curvature two-form $\frac{1}{2} R_{A B \mu \nu} d x^{\mu} \wedge d x^{\nu}$; or as acting wholly on internal Lorentz indices if one 
considers the curvature components of $\frac{1}{2} R_{A B C D} e^{C} \wedge e^{D}$ expressed in vierbein basis. For convenience this latter point of view is adopted here. Given a non-degenerate vierbein, these concepts are interchangeable. The duality operation will be denoted by a tilde over the indices, so $R_{\widetilde{A B C D}} \equiv \frac{1}{2} \epsilon_{A B E F} R^{E F}{ }_{C D}$ implies dualizing on the left and $R_{A B \widetilde{C D}} \equiv \frac{1}{2} \epsilon_{C D E F} R_{A B}^{E F}$ on the right. In the decomposition into self and anti-self-dual projections this mapping $\Lambda_{2}^{ \pm} \mapsto \Lambda_{2}^{ \pm}$can be viewed as a $6 \times 6$ matrix which assumes the form $\left[\begin{array}{cc}S^{+} & C^{+} \\ C^{-} & S^{-}\end{array}\right]$田, 5. In terms of curvature components, the elements of the $3 \times 3$ matrix $S_{a b}^{+}$are

$$
S_{a b}^{+} \equiv+\frac{1}{2}\left(R_{0 a 0 b}+R_{0 a \widetilde{b b}}\right)+\frac{1}{2}\left(R_{\widetilde{0 a} 0 b}+R_{\widetilde{0 a} \widetilde{b b}}\right),
$$

while $S^{-}$and $C^{ \pm}$are defined by replacing the signs in the definition of $S^{+}$above following the prescription

$$
\begin{array}{ll}
S^{+} \sim(+,+,+,+), & S^{-} \sim(+,-,-,-), \\
C^{+} \sim(+,-,+,-), & C^{-} \sim(+,+,-,+) .
\end{array}
$$

Consequently it can be checked that $S^{+}\left(S^{-}\right)$is self-dual (anti-self-dual) with respect to both left and right duality operations, while $C^{+}\left(C^{-}\right)$is self-dual (anti-self-dual) under left duality and anti-self-dual (self-dual) under right duality operations. Furthermore it is known [1] that a metric satisfies Einstein's equations if and only if $C^{ \pm}$vanishes i.e. the $6 \times 6$ matrix above assumes the block diagonal form, and its trace equals $-2 \lambda$. On-shell, the doubly self and anti-self-dual $S^{ \pm}$correspond precisely to the curvature components of the Ashtekar connections [6].

While not all Einstein manifolds possess (anti)self-dual Riemann-Christofel curvatures, it follows from the block diagonal requirement that a manifold is nevertheless Einstein only if the (anti)self-dual part of its spin connection is also doubly (anti)self-dual. So when expressed in terms of (anti)self-dual spin connections $A_{a}^{ \pm}=\omega_{0 a} \pm \frac{1}{2} \epsilon_{0 a b c} \omega^{b c}$, all Einstein manifolds are (anti)instantons in the sense $* F_{a}^{ \pm}= \pm F_{a}^{ \pm}$, with $F_{a}^{ \pm} \equiv d A_{a}^{ \pm}+\frac{1}{2} \epsilon_{0 a b c} A^{ \pm b} \wedge A^{ \pm c}$ ) [7]. Many known exact solutions also have finite Einstein-Hilbert action.

The covariant Samuel-Jacobson-Smolin actions [8] which capture the canonical variables and constraints of Ashtekar can be written as

$$
\mathcal{A}^{ \pm}=\frac{1}{16 \pi G} \int\left(2 F^{ \pm a} \wedge \Sigma_{a}^{ \pm}+\frac{\lambda}{3} \Sigma^{ \pm a} \wedge \Sigma_{a}^{ \pm}\right)
$$

These are just the self and anti-self-dual projections of the Einstein-Hilbert-Palatini action, plus the usual cosmological term written in terms of $\Sigma^{ \pm}$. Remarkably either the + or - action is all one needs to reproduce equations of motion equivalent to Einstein's theory. Note that \pm variables are interchanged under an orientation reversal such as under parity transformation. In this article - i.e. anti-self-dual variables and the corresponding action will be adopted.

The equations of motion which follow from variations of $A_{a}^{-}, e_{0}$ and $e_{a}$ are respectively

$$
\begin{aligned}
D_{A^{-}} \Sigma^{-a} & =0, \\
2 F_{a}^{-} \wedge e^{a}+\frac{\lambda}{3} \epsilon_{0 a b c} e^{a} \wedge e^{b} \wedge e^{c} & =0, \\
2 F_{a}^{-} \wedge e_{0}+2 \epsilon_{0 a b c} e^{b} \wedge F^{-c}+\lambda \epsilon_{0 a b c} e^{0} \wedge e^{b} \wedge e^{c} & =0 .
\end{aligned}
$$

The first equation is solved by $A_{a}^{-}=\omega_{0 a}-\frac{1}{2} \epsilon_{0 a b c} \omega^{b c}$, where $\omega_{A B}$ is the torsionless spin connection respecting $d e_{A}+\omega_{A B} \wedge e^{B}=0$. Since $F_{a}^{-}$is anti-self-dual and $\Sigma_{a}^{-} \wedge \Sigma_{b}^{-}=-2 \delta_{a b}(* 1)$, it is possible to expand $F_{a}^{-}$as

$$
F_{a}^{-}=S_{a b}^{-} \Sigma^{-b}
$$

where $S_{a b}^{-}=-\frac{1}{2}\left(F_{a}^{-} \wedge \Sigma_{b}^{-}\right)$is constrained by

$$
S_{a b}^{-}=S_{b a}^{-} \quad \text { and } \quad \operatorname{Tr}\left(S^{-}\right)=-\lambda
$$

to satisfy the rest of the equations of motion [9,10]. On-shell, $S_{a b}^{-}$is identical to the $3 \times 3$ matrix with the same notation mentioned previously in Eq.(2.2) (see also Eq.(A5) of the Appendix). The Appendix contains a brief proof of the equivalence of this description to Einstein's field equations. There have been suggestions to arrive at a metric-independent description of General Relativity through $\Sigma_{a}^{-}=\left(S^{-}\right)_{a b}^{-1} F^{-b}$ 11. However, the Abelian solutions addressed in this article demonstrate that this cannot be done in general, since for these configurations $S^{-}=\operatorname{diag}(0,0,-\lambda)$ is noninvertible. On the other hand Eq.(2.5) remains valid for all Einstein manifolds with non-degenerate vierbeins. 


\section{ABELIAN INSTANTONS: EXPLICIT EXAMPLES}

The examples can be expressed in terms of four-dimensional polar coordinates $(R, \theta, \phi, \psi)$ with $R^{2} \equiv$ $\sum_{K=0}^{K=3} x^{K} x^{K}, 0 \leq \theta<\pi, 0 \leq \phi<2 \pi, 0 \leq \psi<4 \pi ;$ and

$$
\begin{aligned}
\Theta_{1} & =\frac{1}{2}(\sin \psi d \theta-\sin \theta \cos \psi d \phi), \\
\Theta_{2} & =\frac{1}{2}(-\cos \psi d \theta-\sin \theta \sin \psi d \phi), \\
\Theta_{3} & =\frac{1}{2}(d \psi+\cos \theta d \phi) .
\end{aligned}
$$

$\Theta_{a}$ satisfy $d \Theta_{a}=\epsilon_{a b c} \Theta^{b} \wedge \Theta^{c}$, and $-2 \Theta_{a}$ are the Maurer-Cartan 1-forms on $S^{3}$.

(i) Consider first the complex projective space $\overline{C P}_{2}$ and the well-known Fubini-Study metric

$$
d s^{2}=\frac{(d R)^{2}}{\left(1+\frac{\lambda}{6} R^{2}\right)^{2}}+\frac{\left(R \Theta_{1}\right)^{2}}{\left(1+\frac{\lambda}{6} R^{2}\right)}+\frac{\left(R \Theta_{2}\right)^{2}}{\left(1+\frac{\lambda}{6} R^{2}\right)}+\frac{\left(R \Theta_{3}\right)^{2}}{\left(1+\frac{\lambda}{6} R^{2}\right)^{2}}
$$

The equation of motion $D_{A^{-}} \Sigma_{a}^{-}=0$ implies $A_{a}^{-}=\omega_{0 a}-\frac{1}{2} \epsilon_{a b c} \omega^{b c}$. Choosing the vierbein as $e_{A}=$ $\left\{\frac{d R}{\left(1+\frac{\lambda}{6} R^{2}\right)},-\frac{R \Theta_{1}}{\left(1+\frac{\lambda}{6} R^{2}\right)^{\frac{1}{2}}},-\frac{R \Theta_{2}}{\left(1+\frac{\lambda}{6} R^{2}\right)^{\frac{1}{2}}},-\frac{R \Theta_{3}}{\left(1+\frac{\lambda}{6} R^{2}\right)}\right\}$ leads to

$$
A_{1}^{-}=A_{2}^{-}=0, \quad \text { and } \quad A_{3}^{-}=-\frac{\lambda R^{2} \Theta_{3}}{2\left(1+\frac{\lambda}{6} R^{2}\right)}
$$

giving

$$
F_{1}^{-}=F_{2}^{-}=0, \quad \text { and } \quad F_{3}^{-}=d A_{3}^{-}=-\lambda \Sigma_{3}^{-},
$$

i.e. $F_{a}^{-}=S_{a b}^{-} \Sigma^{-b}$ with $S_{a b}^{-}=\operatorname{diag}(0,0, \lambda)$. The configuration is explicitly Abelian, moreover $* F_{a}^{-}=-F_{a}^{-}$.

(ii) As the next example, take the famous Eguchi-Hanson manifold $\overline{E H}$ with metric

$$
d s^{2}=\left[1-(a / R)^{4}-\frac{\lambda}{6} R^{2}\right]^{-1}(d R)^{2}+\left(R \Theta_{1}\right)^{2}+\left(R \Theta_{2}\right)^{2}+\left[1-(a / R)^{4}-\frac{\lambda}{6} R^{2}\right]\left(R \Theta_{3}\right)^{2} .
$$

Adopting $e_{A}=\left\{\left[1-(a / R)^{4}-\frac{\lambda}{6} R^{2}\right]^{-\frac{1}{2}} d R,-R \Theta_{1},-R \Theta_{2},-\left[1-(a / R)^{4}-\frac{\lambda}{6} R^{2}\right]^{\frac{1}{2}} R \Theta_{3}\right\}$ results in

$$
A_{1}^{-}=A_{2}^{-}=0, \quad \text { and } \quad A_{3}^{-}=-\frac{\lambda}{2} R^{2} \Theta_{3}
$$

and

$$
F_{1}^{-}=F_{2}^{-}=0, \quad \text { and } \quad F_{3}^{-}=-\lambda \Sigma_{3}^{-}
$$

Again $F_{a}^{-}=S_{a b}^{-} \Sigma^{-b}$ with $S_{a b}^{-}=\operatorname{diag}(0,0, \lambda)$ is explicitly Abelian and anti-self-dual with respect to $*$.

It is a matter of convention whether a particular vierbein orientation is associated to $M$ or $\bar{M}$. However it should be mentioned that these projections of the spin connections are orientation dependent. For instance, an orientation reversal under parity transformation $\left(e_{0} \mapsto e_{0}, e_{a} \mapsto-e_{a}\right)$ can produce non-Abelian configurations. This corresponds to looking at $S^{+}$instead. The full Riemannian curvature of Einstein manifolds is contained in $S^{+}$together with $S^{-}$. If one of the projections is an Abelian configuration, then by an appropriate choice of orientation it suffices to consider the case of $S^{-}$for which this is true. Configurations with opposite orientations are not however not related by general coordinate and gauge transformations if they have non-vanishing invariants which are odd under orientation reversal. This is true of the two examples given above. They possess non-vanishing values of the signature invariant [5]. Further discussions on these and related issues for Ashtekar connections can be found in Ref. [10]. 


\section{A THEOREM OF KOBAYASHI AND NOMIZU ON BUNDLE REDUCTION, AND THE HIGGS TRIPLET FOR $S O(3)$}

A powerful theorem of Kobayashi and Nomizu [2] asserts that bundle reduction is predicated on the existence of a covariantly constant field. Proposition 7.4 of Ref. [2] repeated verbatim is:

Let $P(M, G)$ be a principle fiber bundle and $E(M, G / H, G, P)$ the associated bundle with standard fiber $G / H$, where $H$ is a closed subgroup of $G$. Let $\sigma: M \mapsto E$ be a cross section and $Q(M, H)$ the reduced subbundle of $P(M, G)$ corresponding to $\sigma$. Then a connection $\Gamma$ is reducible to a connection $\Gamma^{\prime}$ if and only if $\sigma$ is parallel with respect to $\Gamma$.

The theorem is, remarkably, contemporaneous with, if not earlier than, seminal investigations of symmetry breaking in gauge field theories in particle physics in the 1960s [12]. It also implies, as we shall see, that the bundle reduction is a gauge and diffeomorphism invariant concept. So the Abelian nature of these Einstein manifolds is physically meaningful in General Relativity.

In the previous section Abelian instanton configurations were displayed, despite the gauge group of the $A_{a}^{-}$connection being in general $S O(3)$ in value. By Kobayashi and Nomizu's proposition, a Nambu-Goldstone-Higgs field [12] $\sigma$ must be at work. Let us therefore proceed to demonstrate this below and in the next section. To wit, we specialize to $G=S O(3)$ and $H=S O(2)=U(1)$. Furthermore let $\sigma$ correspond to a Higgs triplet $\phi_{a}(a=1,2,3), \Gamma$ to the connection $A_{\mu a}^{-} d x^{\mu}$, and $\Gamma^{\prime}$ to $A_{\mu a}^{-} \widehat{\phi}^{a} d x^{\mu}$ with $\widehat{\phi}^{a} \equiv \phi^{a} / \sqrt{\phi_{b} \phi^{b}}$. The clause "parallel with respect to $\Gamma^{\prime}$ in the above proposition is now the same as

$$
D_{A^{-}} \phi_{a}=d \phi_{a}+\epsilon_{0 a b c} A^{-b} \wedge \phi^{c}=0
$$

This is a well-known condition characterizing "symmetry breaking" 13]. It is instructive to recall some results which are relevant to symmetry breaking of $S O(3)$ to $U(1)$. Contracting $\phi^{a}$ with Eq. (4.1) results in $d \phi^{2}=d\left(\phi_{a} \phi^{a}\right)=0$. Thus the magnitude of $\vec{\phi}$ is constant, and therefore the allowed values of $\vec{\phi}$ lie on a 2-sphere of constant radius $\sqrt{\phi_{a} \phi^{a}}=K$ in internal space. Regardless of what direction $\vec{\phi}$ is pointing in internal space, it is invariant with respect to Abelian $U(1)$ rotations about the $\widehat{\vec{\phi}}$-axis. This symmetry forms the "little group" $H=U(1)$ of $\vec{\phi}$, while every other point on the 2 -sphere is accessible by an $S O(3) / U(1)$ rotation. Thus the allowed values of $\vec{\phi}$ are parametrized by $G / H$ with $G=S O(3)$. After a little algebra contraction of $\epsilon^{a d e} \phi_{d}$ with Eq.44.1) can be shown to yield (for $K \neq 0$ )

$$
A_{a}^{-}=\left(A_{b}^{-} \widehat{\phi}^{b}\right) \widehat{\phi}_{a}+\epsilon_{0 a b c}\left(d \widehat{\phi}^{b}\right) \widehat{\phi}^{c} .
$$

This shows the decomposition of the $S O(3)$ connection into its Higgs projection and a piece depending only on $\vec{\phi}$. We may then perform in general local (spacetime-dependent) gauge transformations to arrive at the U-gauge 13] in which the Higgs field assumes $\phi^{a}=\phi^{3} \delta_{3}^{a}$. As a result Eq.(4.2) implies $A_{1}^{-}=A_{2}^{-}=0$, and $A_{3}^{-}=\left(A_{3}^{-} \widehat{\phi}^{3}\right) \widehat{\phi}^{3}$ is the only non-vanishing component. A reduction of the connection from $A_{a}^{-}$to Abelian $A_{3}^{-}$has occurred, because $\vec{\phi}$ satisfies the conditions of the theorem as a consequence of Eq. (4.1). It may appear that there could be obstructions, especially for topologically nontrivial configurations, to the existence of a globally defined U-gauge. However, nontrivial configurations -for instance, 't Hooft-Polyakov monopoles associated with symmetry breaking [14]- can be described equally well within the U-gauge [13]. The reason is although more than one coordinate patch is required, the nontrivial topological information is captured by the transition functions between the different patches. These transition functions are therefore $\mathrm{H}$-valued in the U-gauge, and so the reduced bundle and connection are restricted to be $\mathrm{H}$-valued entities while retaining the topological information.

\section{DYNAMICAL BUNDLE REDUCTION AND ORDER PARAMETER FOR THE ABELIAN INSTANTONS}

In the previous section bundle reduction from $S O(3)$ to $U(1)$ was caused by a Higgs triplet obeying Eq.44.1), in accordance with the theorem of Kobayashi and Nomizu. Such a mechanism is at work for Einstein manifolds with $A_{a}^{-}$ Abelian instanton connections. Let us consider the order parameter $S_{a b}^{-}$. As shown, for Abelian instantons it assumes the form $S_{a b}^{-}=\operatorname{diag}(0,0,-\lambda)$. This can be can translated into manifestly gauge-covariant language. Suppose $\vec{\phi}$ has constant magnitude such that $\phi^{2} \equiv \phi^{a} \phi_{a}=\lambda>0$. Then by an $S O(3)$ gauge rotation, the U-gauge configuration $\phi_{a}=-\delta_{a}^{3} \sqrt{\lambda}$ can be transformed into the generic Higgs triplet $\phi_{a}=O_{a b} \phi^{3} \delta_{3}^{b}$ i.e. $\phi_{a}=\left(\phi_{1}, \phi_{2}, \phi_{3}\right)$, and $S_{a b}^{-}$into $\left(O S^{-} O^{T}\right)_{a b}=-\phi_{a} \phi_{b}$. An explicit $S O(3)$ matrix which achieves this purpose is 


$$
O=-\left[\begin{array}{ccc}
\frac{\phi_{3}}{\sqrt{\phi_{1}^{2}+\phi_{3}^{2}}} & \frac{\phi_{1} \phi_{2}}{\left.\sqrt{\phi^{2}\left(\phi_{1}^{2}+\phi_{3}^{2}\right.}\right)} & \frac{\phi_{1}}{\sqrt{\phi^{2}}} \\
0 & \frac{-\sqrt{\phi_{1}^{2}+\phi_{3}^{2}}}{\sqrt{\phi^{2}}} & \frac{\phi_{2}}{\sqrt{\phi^{2}}} \\
-\frac{\phi_{1}}{\sqrt{\phi_{1}^{2}+\phi_{3}^{2}}} & \frac{\phi_{2} \phi_{3}}{\left.\sqrt{\phi^{2}\left(\phi_{1}^{2}+\phi_{3}^{2}\right.}\right)} & \frac{\phi_{3}}{\sqrt{\phi^{2}}}
\end{array}\right] ; \quad \text { with } \quad O O^{T}=O^{T} O=I, \quad \text { and } \quad \operatorname{det}(O)=1 .
$$

Consequently the manifestly covariant form,

$$
S_{a b}^{-}=-\phi_{a} \phi_{b} \quad \text { with } \quad \phi^{2}=\lambda,
$$

is a gauge equivalent description of all the Abelian instanton configurations discussed previously. It also leads to $\phi^{a} F_{a}^{-}=-\lambda \phi^{a} \Sigma_{a}^{-} . S^{-}$has eigenvalues $\left\{0,0,-\phi^{2}=-\lambda\right\}$, and satisfies the contraints of General Relativity by being symmetric and with trace $-\lambda$.

Let us further check that the symmetry reduction condition, Eq.(4.1), is indeed true. It is worthwhile to recall that Eq.(4.1) is gauge and diffeomorphism invariant, so it suffices to verify that the equation holds for a particular gauge of convenience. In the U-gauge, $S^{-}=\operatorname{diag}\{0,0,-\lambda\}$. Through $F_{a}^{-}=S_{a b}^{-} \Sigma^{-b}$ we obtain $F_{1,2}^{-}=0$ and $F_{3}^{-}=-\lambda \Sigma_{3}^{-} \neq 0$. These are solved by $A_{1,2}^{-}=0$ and $F_{3}^{-}=d A_{3}^{-}=-\lambda \Sigma_{3}^{-}$. With $A_{a}^{-}=\left(0,0, A_{3}^{-}\right)$, the condition $D_{A^{-}} \phi_{a}=0$ reduces in component form to

$$
\begin{aligned}
d \phi_{1}-A_{3}^{-} \wedge \phi_{2} & =0 \\
d \phi_{2}+A_{3}^{-} \wedge \phi_{1} & =0 \\
d \phi_{3} & =0 .
\end{aligned}
$$

These equations are clearly satisfied by $\phi_{a}=(0,0,-\sqrt{\lambda})$ in the U-gauge, and hence for all gauges and coordinate choices. It is thus correct to associate Einstein manifolds described by such Abelian instantons as bundle reductions of $S O(3)$ to $U(1)$, and $S^{-}$as the order parameter which characterize them by Eq.(5.2). Note that with $S_{a b}^{-}=-\phi_{a} \phi_{b}$ the consistency condition of the Bianchi identity,

$$
D_{A^{-}} F_{a}^{-}=\left(D_{A^{-}} S\right)_{a b} \wedge \Sigma^{-b}+S_{a b}\left(D_{A^{-}} \Sigma\right)^{-b}=0,
$$

is ensured because $D_{A^{-}} \Sigma^{-a}=0$ and $D_{A^{-}} \phi_{a}=0$.

It is to be noted that $S^{-}$is itself a composite of the vierbien through $S_{a b}^{-}=-* \frac{1}{2}\left(F_{a}^{-} \wedge \Sigma_{b}^{-}\right)$with $F_{a}^{-}$being, on-shell, the anti-self-dual part of the curvature of the torsionless spin connection. Furthermore the Higgs field $\phi_{a}$ can be gauge-transformed away, apart from a non-vanishing third component which is associated with the cosmological constant through $\phi_{3}=-\sqrt{\lambda}$.

These Einstein manifolds with non-vanishing $\lambda$ demonstrate that gravitational self-interactions can generate this mechanism of bundle reduction dynamically, despite the absence of additional fundamental scalar fields and other physical matter. As a corollary, we note that the (anti)self-dual part of the spin connections of Einstein manifolds can be Abelian instantons only if the cosmological constant is non-zero, otherwise the condition $F_{a}^{-}=S_{a b}^{-} \Sigma^{-b}$ cannot be simultaneously satisfied for $\operatorname{Tr}\left(S^{-}\right)=-\lambda=0$ and $S^{-}=\operatorname{diag}(0,0,-\lambda \neq 0)$ for Abelian configurations.

\section{ABELIAN INSTANTONS AND KÄHLER-EINSTEIN MANIFOLDS}

The Fubini-Study and Eguchi-Hanson explicit solutions displayed in Section III are also known to be Kähler-Einstein manifolds [5]. This can be generalized to other Abelian solutions. When $S^{-}$is gauge equivalent to $\operatorname{diag}(0,0,-\lambda)$, the anti-self-dual part of the spin connection is described by an Abelian instanton $A_{a}^{-}=\left(0,0, A_{3}^{-}\right)$. It follows that $F_{a}^{-}=S_{a b}^{-} \Sigma^{-b}$ reduce to

$$
F_{1,2}^{-}=0, \quad F_{3}^{-}=d A_{3}^{-}=-\lambda \Sigma_{3}^{-} .
$$

Furthermore the equations of motion $\left(D_{A^{-}} \Sigma^{-}\right)^{a}=0$ become

$$
\begin{aligned}
& d \Sigma_{1}^{-}-A_{3}^{-} \wedge \Sigma_{2}^{-}=0, \\
& d \Sigma_{2}^{-}+A_{3}^{-} \wedge \Sigma_{1}^{-}=0,
\end{aligned}
$$




$$
d \Sigma_{3}^{-}=0 .
$$

Let the complex one-forms $\Omega^{1,2}$ be defined as $\Omega^{1} \equiv-e^{0}+i e^{3}$ and $\Omega^{2} \equiv e^{1}+i e^{2}$. The metric can then be expressed as

$$
d s^{2}=e_{A} e^{A}=g_{\mathcal{A B}} \Omega^{\mathcal{A}} \bar{\Omega}^{\mathcal{B}},
$$

with $g_{\mathcal{A B}}=\delta_{\mathcal{A B}}(\mathcal{A}, \mathcal{B}$ assume values 1 to 2$)$ being explicitly Hermitian. Furthermore, the Kähler form, which is real, can be identified to be

$$
\Sigma_{3}^{-}=-e^{0} \wedge e^{3}+e^{1} \wedge e^{2}=\frac{i}{2} \Omega^{\mathcal{A}} \wedge \bar{\Omega}^{\mathcal{A}} .
$$

A metric is said to be a Kähler if the Kähler form is closed. But it follows from Eq.(6.2) that this is indeed the case. So Einstein manifolds with $A^{-}$which are Abelian instantons can be identified as Kähler-Einstein manifolds.

\section{ACKNOWLEDGMENTS}

The research for this work has been supported in part by funds from the National Center for Theoretical Sciences, and the Physics Department of the National Cheng Kung University, Taiwan. This article is an extended version of a talk presented by the author at the International Workshop on Geometric Physics (July 2000) held in Hsinchu, Taiwan, at the National Center for Theoretical Sciences.

\section{APPENDIX A: EQUIVALENCE TO EINSTEIN'S FIELD EQUATIONS}

Let us briefly show that

$$
D_{A}^{-} \Sigma^{-a}=0
$$

and

$$
F_{a}^{-}=S_{a b}^{-} \Sigma^{-b},
$$

with

$$
\epsilon_{0 a b c} S^{-b c}=0 \text { and } \operatorname{Tr}\left(S^{-}\right)=-\lambda
$$

are equivalent to Einstein's field equations.

Eq.(A1) is uniquely solved by $A_{a}^{-}=\omega_{0 a}-\frac{1}{2} \epsilon_{0 a b c} \omega^{b c}$, from which $F_{a}^{-}=R_{0 a}-\frac{1}{2} \epsilon_{0 a b c} R^{b c}$, where $R_{A B}=d \omega_{A B}+$ $\omega_{A}^{C} \wedge \omega^{C}{ }_{B}$. The rest of Eqs.(2.4) hold on substituting $F_{a b}^{-}=S_{a b}^{-} \Sigma^{-b}$ provided $S^{-}$is symmetric and of trace $-\lambda$. However,

$$
\begin{aligned}
F_{a}^{-} & =\frac{1}{2}\left(R_{0 a A B}-\frac{1}{2} \epsilon_{0 a b c} R_{A B}^{b c}\right) e^{A} \wedge e^{B} \\
& =S_{a b}^{-}\left(-e^{0} \wedge e^{b}+\frac{1}{2} \epsilon_{0 a b c} e^{b} \wedge e^{c}\right)
\end{aligned}
$$

implies

$$
S_{a b}^{-}=R_{\widetilde{0 a} 0 b}-R_{0 a 0 b}=R_{0 a \widetilde{0 b}}-R_{\widetilde{0 a} \widetilde{b b}}
$$

These lead, together with the constraint $S_{a b}^{-}=S_{b a}^{-}$, to

$$
R_{\widetilde{0 a} 0 b}=R_{0 a \widetilde{0 b}} \text { and } R_{0 a 0 b}=R_{\widetilde{0 a} \widetilde{0 b}} .
$$

It can then be shown these are equivalent to

$$
R_{\widetilde{A B} \widetilde{C D}}=R_{A B C D}
$$


and $\operatorname{Tr}\left(S^{-}\right)=-\lambda$ implies the Ricci scalar

$$
R_{A B}{ }^{A B}=R=4 \lambda .
$$

It is known 15] that Eqs.(A7-A8) are equivalent to Einstein's equations $R_{\mu \nu}=\lambda g_{\mu \nu}$.

$\dagger \quad$ Electronic Address: cpsoo@mail.ncku.edu.tw

[1] S. Perlmutter et al, Bull. Am. Astron. Soc. 29 (1997) 1351; S. Perlmutter et al, Nature 391 (1998) 51; P. M. Garnavich et al, Astrophys. J. 493 (1998) L53; B. P. Schmidt et al, Astrophys. J. 507 (1998) 46; A. G. Reiss et al, Astron. J. 116 (1998) 1009; S. Perlmutter et al, Astrophys. J. 517 (1999) 565.

[2] S. Kobayashi and K. Nomizu, Chapter II (Theory of Connections), Section 7, Foundations of Differential Geometry, Vol. 1 (John Wiley and Sons, 1963).

[3] G. W. Gibbons and S. W. Hawking (eds.) Euclidean Quantum Gravity (Singapore: World Scientific, 1993) and references therein.

[4] M. F. Atiyah, N. J. Hitchin and I. M. Singer, Proc. Roy. Soc. Lond. A362 (1978) 425.

[5] T. Eguchi, P. B. Gilkey and A. J. Hanson, Phys. Rep. 66 (1980) 213.

[6] A. Ashtekar, Phys. Rev. Lett. 57 (1986) 2244; Phys. Rev. D36 (1986) 1587; New perspectives in canonical gravity, (Bibliopolis, Naples, 1988); Lectures on nonperturbative canonical gravity (Singapore: World Scientific, 1991).

[7] C. Soo, Phys. Rev. D52 (1995) 3484.

[8] J. Samuel, Pramāna J. Phys. 28 (1987) L429; Class. Quantum Grav. 5 (1988) L123; T. Jacobson and L. Smolin, Phys. Lett. B196 (1987) 39, Class. Quantum Grav. 5 (1988) 583.

[9] L. N. Chang and C. Soo, Phy. Rev. D46 (1992) 4547.

[10] L. N. Chang and C. Soo, hep-th/9207056, L. N. Chang and C. Soo, Phys. Rev. D53 (1996) 5682.

[11] R. Capovilla, J. Dell and T. Jacobson, Phys. Rev. Lett. 63 (1989) 2325; R. Capovilla, J. Dell and T. Jacobson, Class. Quantum Grav. 5 (1988) 583.

[12] Y. Nambu, Phys. Rev. Lett. 4 (1960) 380; J. Goldstone, Nuovo Cim. 19 (1961) 15; J. Goldstone, A. Salam and S. Weinberg, Phys. Rev. 127 (1962) 965; P.W. Higgs, Phys. Lett. 12 (1964) 132, Phys. Rev. 145 (1966) 1156; T. W. B. Kibble, Phys. Rev. 155 (1967) 1554.

[13] See, for instance, P. Goddard and D. I. Olive, Rept. Prog. Phys. 41 (1978) 1357.

[14] G. 't Hooft, Nucl. Phys. B79 (1974) 276; A. M. Polyakov, JETP Lett. 20 (1974) 194, JETP 41 (1976) 988.

[15] See, for instance, F. de Felice and C. J. S. Clarke, Relativity on curved manifolds (Cambridge: Cambridge University Press, 1990). 